\title{
Modificación de las texturas y de los índices de embutibilidad de chapas de aleaciones de aluminio Al 1050 y Al-Mg 5754 mediante laminación asimétrica
}

\author{
M. GARCÍA DE LOMANA, A. RODRÍGUEZ¹, J. TAMAYO', C. ZUBILLAGA², C. GARCÍA-ROSALES², J. GIL SEVILLANO² \\ ${ }^{1}$ Campus Tecnológico Universidad de Navarra (TECNUN), 20009 San Sebastián \\ ${ }^{2}$ Centro de Estudios e Investigaciones Técnicas de Guipúzcoa (CEIT), 20009 San Sebastián
}

\begin{abstract}
El valor del índice $r$ de chapas de aluminio es bajo debido a la textura obtenida tras los procesos convencionales de laminación y recocido. Un incremento de la componente $<111>$ / / DN da lugar a un aumento del índice $r$ en metales bcc y también fcc. Sin embargo, la textura $<111>/ / D N$ no puede producirse en éstos últimos a partir de procesos convencionales de laminación y recocido, pero esta orientación constituye una de las componentes principales de la textura a cortadura en metales fcc. Por tanto, la introducción de deformación a cortadura paralela al plano de laminación durante el proceso de laminación del aluminio conducirá a una mejora de la embutibilidad. En este trabajo se han estudiado las texturas y los índices $\overline{\mathrm{r}}$ y $\Delta r$ de chapas de $\mathrm{Al} 1050$ y Al-Mg 5754 tras laminación asimétrica. La laminación se ha realizado con los rodillos dispuestos de tal manera que uno gira al doble de velocidad que el otro. Se ha conseguido aumentar fuertemente la componente $<111>/ /$ DN respecto a la textura de laminación convencional, lo cual supone un incremento significativo del índice $\overline{\mathrm{r}}$, aunque el valor de $\Delta r$ es grande. Esta textura se puede retener tras recocidos de restauración.
\end{abstract}

Palabras clave: laminación asimétrica; chapas de aluminio; deformación a cortadura; textura; indicer

\section{Modification of textures and drawability values of sheets of aluminum alloys al 1050 and al-mg 5754 by means of asymmetric rolling}

The $r$-value of aluminum sheets is low because of the texture obtained after conventional rolling and annealing processes. An increase of the $<111>/$ / ND component results in an increment of the $r$-value not only in bcc metals, but also in metals with fcc structure. However, the $<111>$ / / ND texture cannot be obtained in fcc metals by a conventional rolling and annealing process, but this orientation is one of the main components of the shear texture in fcc metals. Consequently, the introduction of shear deformation parallel to the rolling plane during the aluminum rolling procedure will result in an improvement of its drawability. In this study the texture as well as the $\overline{\mathrm{r}}$ and $\Delta r$-values of sheets of $\mathrm{Al} 1050$ and $\mathrm{Al}-\mathrm{Mg} 5754$ has been investigated after asymmetric rolling. The rolling process was performed with the rolls arranged in such a way that one rotates a double velocity than the other one. A considerable increase of the $<111>$ / / ND component with respect to the texture of conventional rolling has been achieved, resulting in a significant increase of the $\overline{\mathrm{r}}$-value, even though the $\Delta r$-value is large. This texture can be retained by recovering annealing.

Keywords: asymmetric rolling; aluminum sheets, shear deformation; texture; r-value

\section{INTRODUCCIÓN}

El valor del índice de embutibilidad $r$ de chapas de aluminio y sus aleaciones es bajo debido a la textura obtenida tras los procesos convencionales de laminación y recristalización. Un incremento de la componente $\langle 111\rangle / / \mathrm{DN}$ da lugar a un aumento del índice $r$, no sólo en metales bcc sino también en aquellos con estructura fcc. La textura $\langle 111\rangle / /$ DN no puede producirse en metales fcc a partir de procesos convencionales de laminación y recristalización, pero esta orientación constituye una de las componentes principales de la textura a cortadura en metales fcc. Por tanto, la introducción de deformación a cortadura paralela al plano de laminación durante el proceso de laminación del aluminio tendrá como consecuencia una mejora de la embutibilidad. Recientemente se ha demostrado que es posible conseguir dicha textura de cortadura mediante laminación asimétrica $(1,2)$.

El objetivo del presente trabajo es incrementar la componente $\langle 111\rangle$ / / DN de la textura de un material fcc como el aluminio mediante laminación asimétrica en frío, respecto a la textura que se obtiene mediante laminación convencional. Este incremento implicaría una mejora del índice de embutibilidad, $r$, del aluminio. Dicha mejora se podría explotar tecnológicamente siempre que el nuevo proceso no aumentara excesivamente el índice de anisotropía plana, $\Delta r$.

\section{PARTE EXPERIMENTAL}

Los materiales investigados han sido las aleaciones Al 1050 y Al-Mg 5754. Las chapas de partida de los dos materiales, de $4 \mathrm{~mm}$ de grosor, fueron sometidas previamente a un tratamiento de recristalización a $365^{\circ} \mathrm{C}$ durante una hora (3). Antes y después del tratamiento se midió la dureza HRH (punzón $1 / 8^{\prime \prime}$, carga $60 \mathrm{~kg}$ ), obteniéndose tras el tratamiento una reducción de dureza considerable para el $\mathrm{Al}$, pero no así para el $\mathrm{Al}-\mathrm{Mg}$, como se muestra en la tabla 1. Esto indica que en el Al ha tenido lugar la recristalización total, llegando posiblemente al crecimiento de grano, mientras que en el Al-Mg el material apenas ha recristalizado. Las chapas sometidas a este tratamiento térmico son las que se han utilizado como material de partida para la laminación asimétrica.

La laminación se ha realizado con los rodillos conectados a engranajes de distinto radio, de manera que uno de los rodillos gira al doble de velocidad que el otro. Se introduce así una fuerte componente de deformación a cortadura en la cara en contacto con el rodillo que gira a mayor velocidad (véase figura 1). Con el fin de producir una deformación a cortadura uniforme en todo el espesor de la chapa, la reducción total se ha realizado acumulando cuatro pasadas sucesivas, en cada una de las cuales se ha alternado la cara de la chapa en contacto 
con el rodillo que gira a mayor velocidad. En cada una de las pasadas la reducción de espesor ha sido del $50 \%$, de manera que el espesor final de la chapa ha sido de $0,25 \mathrm{~mm}$ y la reducción total tras laminación asimétrica del 93\%. La laminación se realizó a temperatura ambiente, utilizando lubricante para evitar el arrugamiento de la chapa.

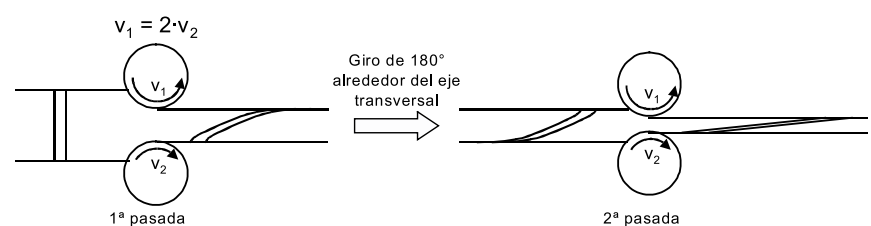

Figura 1: Esquema del proceso de laminación asimétrica para obtener deformación a cortadura (4 pasadas con giro de la chapa de $180^{\circ}$ alrededor del eje transversal entre cada pasada).

Las chapas laminadas asimétricamente fueron sometidas a tratamientos de restauración y recristalización $\left(225^{\circ} \mathrm{C}, 1 \mathrm{~h}\right.$ mantenimiento; y $365^{\circ} \mathrm{C}, 1 \mathrm{~h}$, respectivamente, para los dos materiales (3)).

Antes y después de la laminación y de cada tratamiento se midieron las figuras de polos (111), (200), (220) y (311) en un goniómetro de texturas de rayos- $X$ en modo reflexión, utilizando radiación $\mathrm{Cr}$, Co ó $\mathrm{Cu}-\mathrm{K} \alpha$ filtrada. Estas figuras fueron utilizadas para recalcular las figuras de polos completas con el software MTM-FHM (4). Mediante un modelo de Taylor para plasticidad de policristales, incluido en el citado software, se ha calculado el índice $r$ y la anisotropía plana $\Delta r$ a partir de las texturas medidas.

TABla 1: Dureza HRH (PUnZÓn 1/8", CARGa 60 kg) DE lOS DOS MATERIALES ESTUDIADOS ANTES (SUMINISTRO) Y DESPUÉS DEL TRATAMIENTO DE RECRISTALIZACIÓN A $365^{\circ} \mathrm{C}$ DURANTE $1 \mathrm{~h}$.

\begin{tabular}{|l||c|c|}
\hline & Al 1050 & Al-Mg 5754 \\
\hline \hline Suministro & 76,1 & 92,3 \\
\hline Recristalización & 24,3 & 82,7 \\
\hline
\end{tabular}

\section{RESULTADOS Y DISCUSIÓN}

En las figuras 2 y 3 se muestran las figuras de polos (111) y (200) recalculadas para el Al 1050 y para el Al-Mg 5754 respectivamente antes y después de la laminación asimétrica y de los tratamientos térmicos. En el Al 1050 se observa que, tras el tratamiento de recristalización del material de suministro, la textura adquiere una clara componente cubo, indicando la completa recristalización del material, en concordancia con los valores de dureza medidos (véase tabla 1). En el Al-Mg, sin embargo, la textura apenas se ve alterada tras dicho tratamiento, lo que pone de manifiesto que el material no ha sufrido recristalización. Tras la laminación asimétrica, la figura de polos (111) del Al 1050 muestra un polo de gran intensidad cercano a la orientación $\langle 111\rangle / / \mathrm{DN}$, que es una de las componentes principales de la textura a cortadura. Esta textura se mantiene casi inalterada tras el tratamiento de restauración, pero se transforma en textura con fuerte componente cubo tras el tratamiento de recristalización. En el caso del $\mathrm{Al}-\mathrm{Mg}$, la textura tras la laminación asimétrica muestra asimismo un polo cercano a la orientación $\langle 111\rangle / / \mathrm{DN}$, pero no tan acentuado como en el $\mathrm{Al}$, observándose que la textura previa a la laminación prevalece. Al igual que en el $\mathrm{Al}$ 1050, la textura adquiere también una fuerte componente cubo tras el tratamiento de recristalización.

En la figura 4 se muestra la evolución del índice de embutibilidad promedio $\bar{r}$ y de la anisotropía plana $\Delta r$ para el $\mathrm{Al} 1050$ (izda.) y para el Al-Mg 5754 (drcha.) antes y después de la laminación asimétrica y de
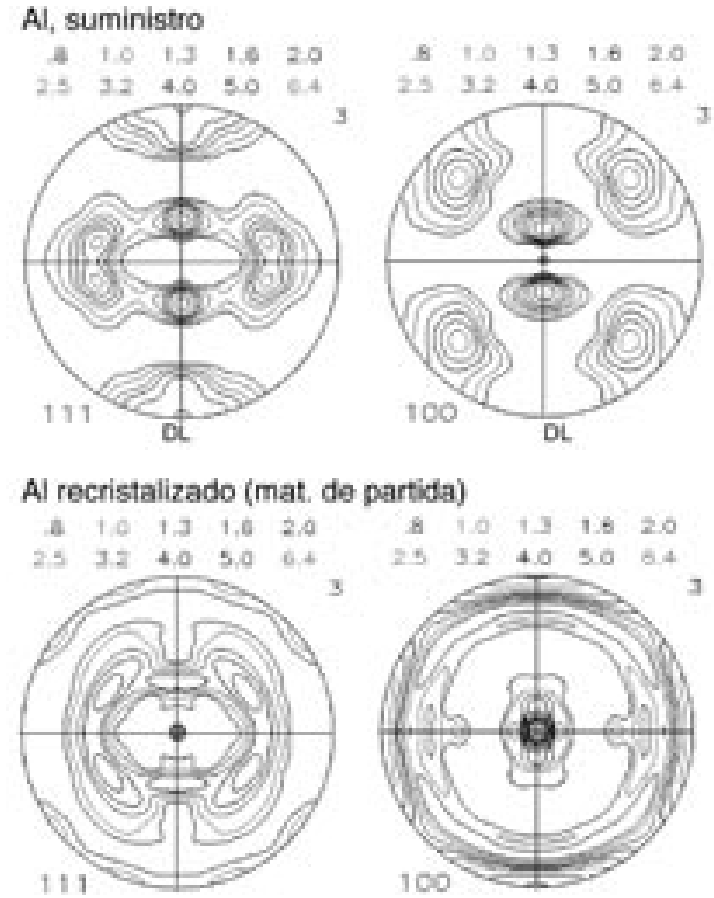

Al, $93 \%$ lam. asimétrica
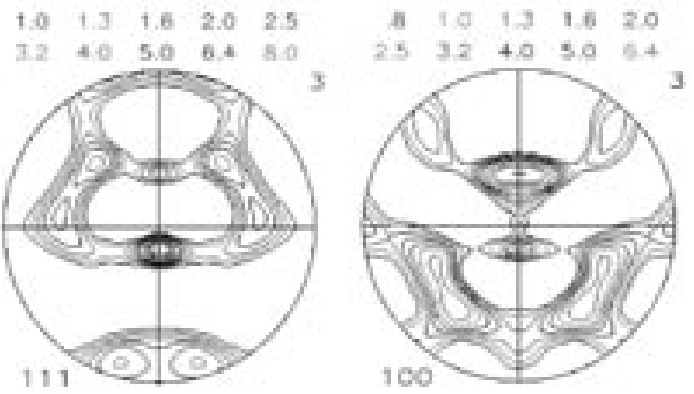

Al lam. asim., restaurado

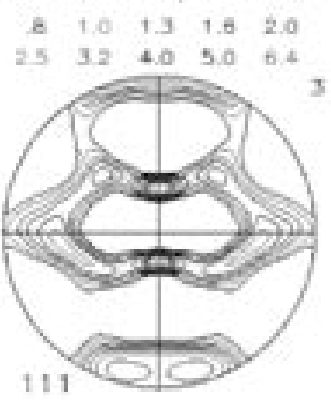

$\begin{array}{lllll}3 & 1.0 & 13 & 1.6 & 20\end{array}$
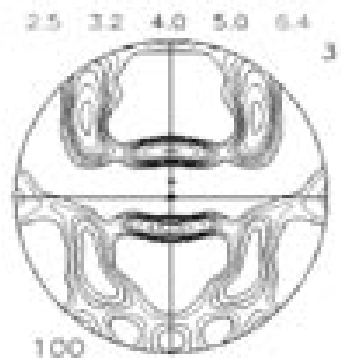

Al lam. asim., recristalizado

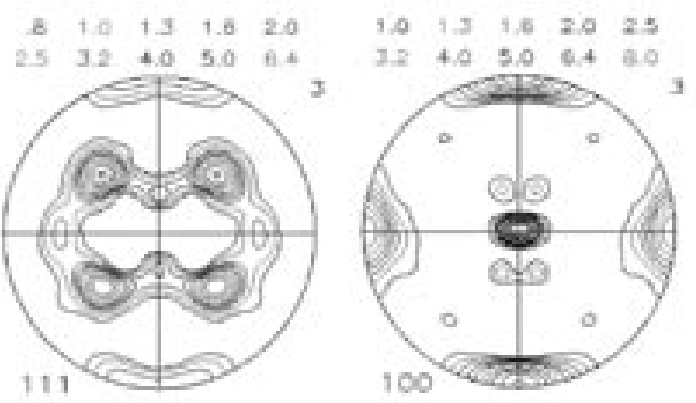

Figura 2: Figuras de polos (111) y (200) recalculadas del Al 1050 antes y después de la laminación asimétrica y tras tratamientos térmicos. 


\section{Al-Mg, suministro}
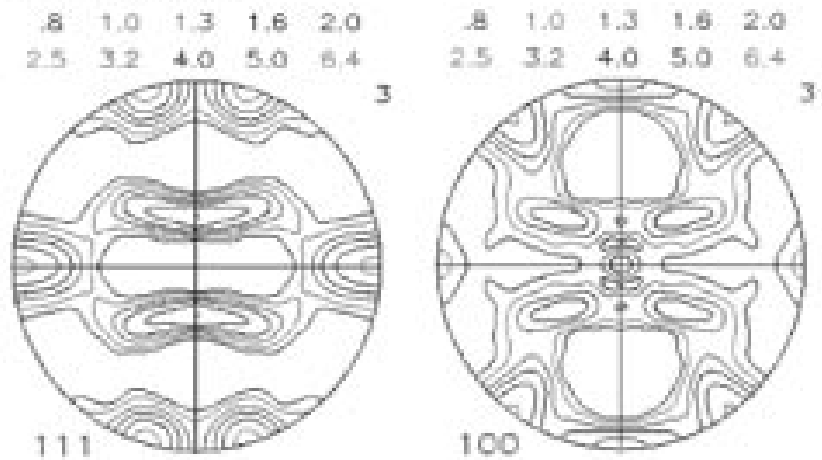

Al-Mg recristalizado (mat. de partida)
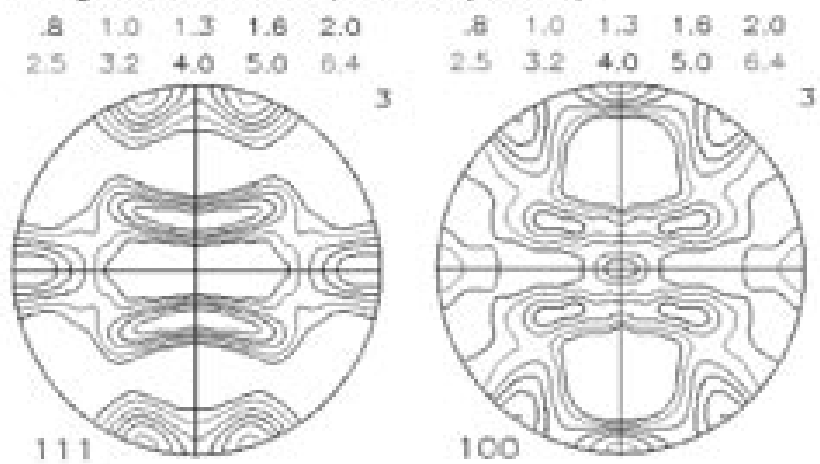

Al-Mg, 93\% lam, asimétrica

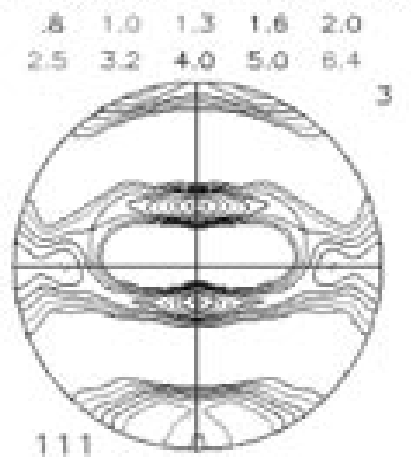

$\begin{array}{lllllll}8 & 1.0 & 1.3 & 1.6 & 2.0\end{array}$
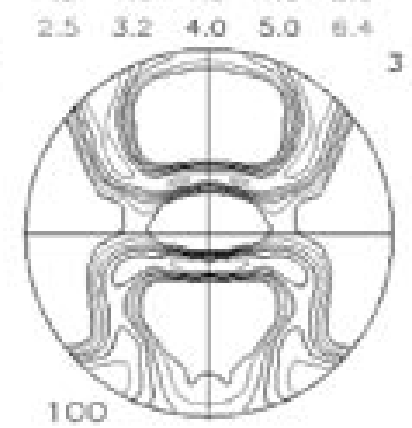

Al-Mg lam. asim., recristalizado

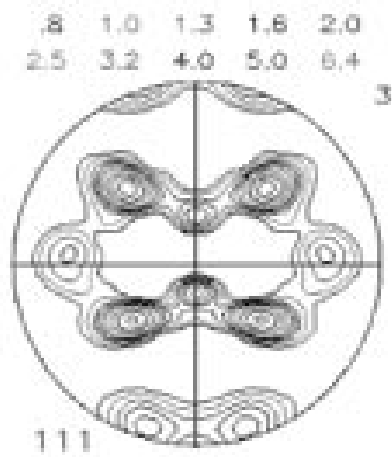

$\begin{array}{llllll}1.0 & 1.3 & 1.6 & 2.0 & 2.5\end{array}$
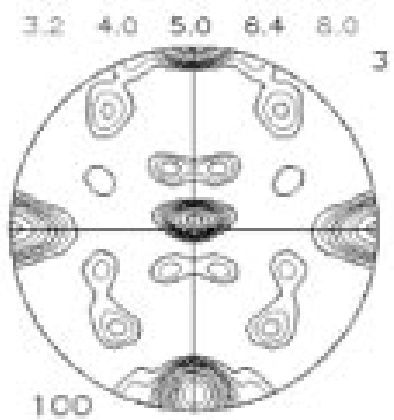

Figura 3: Figuras de polos (111) y (200) recalculadas del Al-Mg 5754 antes y después de la laminación asimétrica y tras tratamientos térmicos.
Al

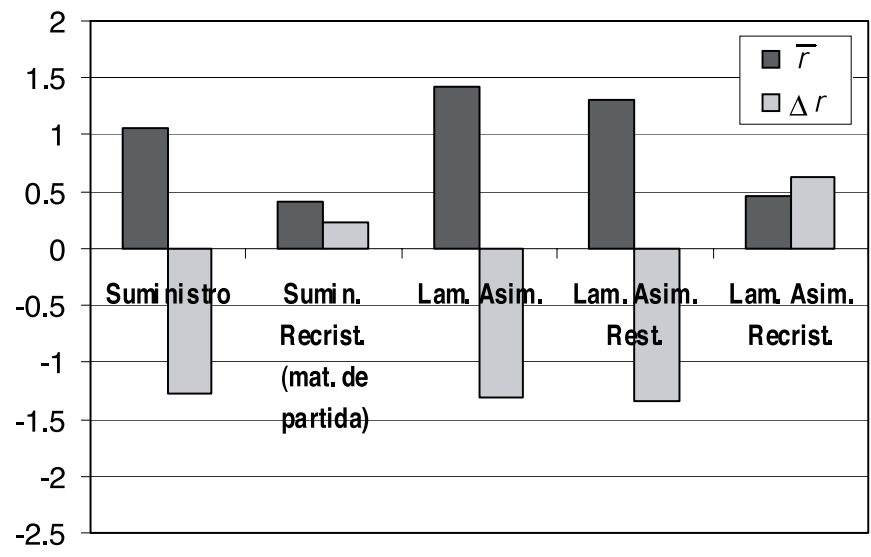

Al-Mg

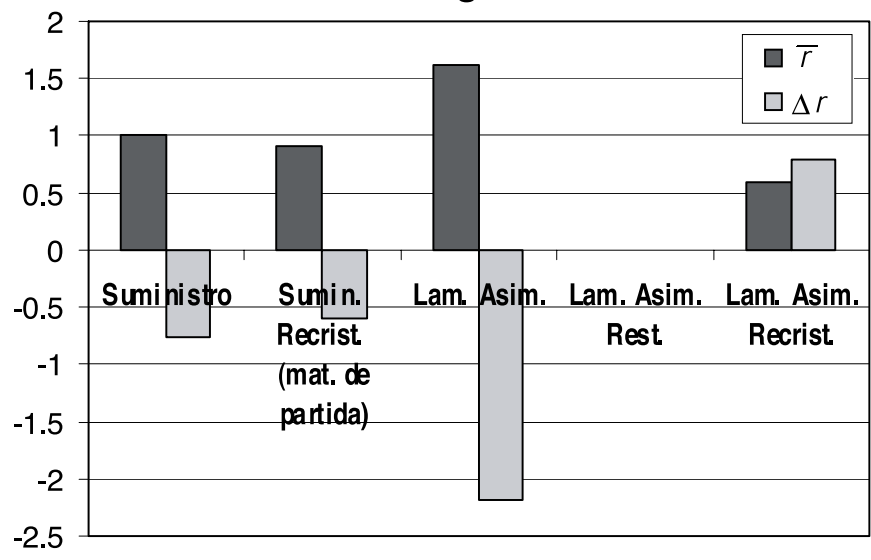

Figura 4: Evolución de los valores de $\mathrm{r}^{-} \mathrm{y} \Delta r$ en chapas de Al 1050 (izda.) y Al-Mg 5754 (drcha.) antes y después de la laminación asimétrica y tras tratamientos térmicos.

los tratamientos térmicos. Los dos materiales en estado de suministro muestran valores de $\overline{\mathrm{r}}$ alrededor de 1 , y de $\Delta r$ entre $-1,3$ y $-0,8$. Tras el tratamiento de recristalización previo a la laminación los valores de $\overline{\mathrm{r}}$ y $\Delta r$ del Al-Mg apenas cambian, mientras que los del $\mathrm{Al}$ disminuyen en módulo considerablemente, en concordancia con las observaciones de las figuras de polos. Tras la laminación asimétrica, el valor de $\overline{\mathrm{r}}$ aumenta notablemente en ambos casos en relación con el material de suministro, obteniéndose valores de 1,4 para el Al y de 1,6 para el Al-Mg. Este incremento supone una mejora significativa en la embutibilidad. Sin embargo, no se observa mejora en el valor de la anisotropía plana $\Delta r$ en ninguno de los materiales, obteniéndose para el $\mathrm{Al}-\mathrm{Mg}$ un valor notablemente mayor en módulo que en el material de suministro, lo cual tendrá como resultado la formación de orejas. Los valores de $\bar{r}$ y $\Delta r$ se mantienen tras el tratamiento de restauración (sólo medido para el $\mathrm{Al}$ ), pero no así tras el de recristalización: la aparición de textura cubo tiene como consecuencia una fuerte reducción de $\bar{r}$.

A la vista de estos resultados, y comparando con los resultados de la bibliografía $(1,2)$, se ve necesario profundizar en la investigación para optimizar el proceso, con el fin de reducir el módulo de $\Delta r$ a la vez que se incrementa el valor de $\bar{r}$. Dicha investigación deberá incluir la caracterización detallada de las microestructuras obtenidas. Los parámetros a investigar en futuros trabajos serían la textura de partida antes de la laminación, la temperatura de laminación asimétrica, la velocidad de los rodillos y la relación de velocidades entre ellos, así como los parámetros ideales de los tratamientos de restauración y recristalización para conseguir que se mantenga la textura deseada. 


\section{CONCLUSIONES}

Las conclusiones del presente trabajo se resumen a continuación:

- Mediante laminación asimétrica, conseguida por accionamiento a distinta velocidad de rotación de los cilindros de trabajo, es posible modificar la textura de chapas de aluminio, aumentando fuertemente la componente $\langle 111\rangle /$ / DN respecto a la textura de laminación convencional.

- La nueva textura supone un incremento significativo del índice de anisotropía promedio, $\overline{\mathrm{r}}$, aunque el valor de la anisotropía plana, $\Delta r$, es grande.

- Esa textura, ventajosa para la embutición, se puede retener tras recocidos de ablandamiento por restauración, pero se pierde si en el recocido se produce recristalización.

- Para explotar adecuadamente los beneficios técnicos de la laminación asimétrica, es necesario optimizar los parámetros del proceso de laminación y recocido con un estudio sistemático.

\section{AGRADECIMIENTOS}

Los autores agradecen a Olivier Bouaziz (IRSID, Maizières-lesMetz, Francia) la sugerencia de cómo modificar fácilmente la velocidad de los cilindros de laminación del laminador de laboratorio utilizado.

\section{BIBLIOGRAFÍA}

1. T. Sakai, S. Hamada, Y. Saito. "Improvement of the r-value in 5052 aluminum alloy sheets having through-thickness shear texture by 2-pass single-roll drive unidirectional shear rolling”. Scripta Mater. 44, 2569-2573 (2002).

2. K.-H. Kim, D.N. Lee. "Analysis of deformation textures of asymmetrically rolled aluminum sheets" Acta Mater. 49, 2583-2595 (2001).

3. Heat treater's guide. Practice and procedures for nonferrous alloys. ASM (1996).

4. P. van Houtte. "The MTM-FHM software system Version 2", Katholieke Universiteit Leuven, Bélgica (1995).

Recibido: 1.2 .03

Aceptado: 30.11 .03 